Revue

Revue de l'histoire des religions

de Ihistoire des religions

\title{
Firmico Materno, L'errore delle religioni pagane
}

Introduzione, traduzione e note a cura di Ennio Sanzi, Roma, Città Nuova Editrice, 2006, 204 p., $21 \mathrm{~cm}$ ("Collana di testi patristici", 191), $18 €$.

\section{Giovanni Tosetti}

\section{OpenEdition}

Journals

Édition électronique

URL : http://journals.openedition.org/rhr/7238

DOI : $10.4000 /$ rhr.7238

ISSN : $2105-2573$

Éditeur

Armand Colin

Édition imprimée

Date de publication : 1 avril 2009

Pagination : 261-264

ISBN : 978-2200-92590-1

ISSN : 0035-1423

\section{Référence électronique}

Giovanni Tosetti, «Firmico Materno, L'errore delle religioni pagane », Revue de l'histoire des religions [En ligne], 2 | 2009, mis en ligne le 12 janvier 2010, consulté le 22 septembre 2020. URL : http:// journals.openedition.org/rhr/7238; DOl : https://doi.org/10.4000/rhr.7238 


\section{COMPTES RENDUS}

Firmico MAterno, L'errore delle religioni pagane, Introduzione, traduzione e note a cura di Ennio SANZI, Roma, Città Nuova Editrice, 2006, 204 p., $21 \mathrm{~cm}$ (“Collana di testi patristici”, 191), $18 €$.

Comme il est de tradition pour la «Collana di testi patristici », dans le présent volume la traduction en langue italienne (p. 69-180) de l'œuvre de Firmicus Maternus s'ouvre par une riche introduction (p. 5-63 ; complétée par une brève indication des éditions disponibles du texte et des traductions, p. 65-66), qui prend en considération surtout les enjeux de Firmicus, les circonstances extra-discursives de composition et l'unité structurelle du De errore. La traduction est accompagnée de notes de commentaire et l'ouvrage se termine par de très utiles indices (des noms et des choses, p. 183-199; des sources, p. 200-201; général, p. 203-204). Tout aussi caractéristique de la collection est la traduction qui se distingue par sa clarté et vise un large public savant. Ce qui est moins typique - et donc particulièrement significatif de la perspective du travail - est le domaine de compétence scientifique de l'auteur, puisqu'Ennio Sanzi [désormais ES] est un historien des religions, spécialiste des phénomènes religieux du second hellénisme. Son approche, relevant principalement des intérêts et de la méthode qui ont caractérisé l'œuvre de U. Bianchi et marquent les travaux de ses disciples (aux volumes signalés par l'auteur à p. 27, n. 45, on peut ajouter ici G. Sfameni Gasparro, An Introduction : Ugo Bianchi and the History of Religions. Some Preliminary Remarks on a Method of Religious-Historical Research, in Ead., Themes and Problems of the History of Religions in Contemporary Europe. Proceedings of the International Seminar [Messina, March 30-31, 2001], Cosenza, Giordano, 2002, p. 19-30), se révèle notamment (comme on le verra) dans les notes du commentaire. Dans le domaine italophone, ES se signale comme le premier à privilégier cette perspective. En effet, l'importante édition commentée de Agostino Pastorino (De errore profanarum religionum, Firenze, La Nuova Italia, 1956), sans oublier l'intérêt historico-religieux, présentait une remarquable attention à l'étude littéraire et notamment rhétorique.

L'intérêt historico-religieux du pamphlet de Firmicus repose sur la nature même de l'œuvre. Comme ES le signale dès le début de son introduction (p. 5), le De errore nous a transmis d' "autentici hapax » sur les cultes païens répandus dans l'Empire romain. En effet, cette œuvre est bien souvent la source littéraire la plus convoquée pour les cultes 
dits « orientaux », selon la dénomination actuelle, qui s’écarte de celle de « religions orientales » fondée sur l'étude séminale de Franz Cumont (Les religions orientales dans le paganisme romain, Paris, Leroux, 1906; voir Giulia Sfameni Gasparro, Misteri e teologie. Per la storia dei culti mistici e misterici nel mondo antico, Cosenza, Giordano, 2003, p. 12, n. 11).

L'introduction d'ES, tout en étant largement tributaire des pages qui ouvrent l'importante édition de Robert Turcan (Firmicus Maternus. L'erreur des religions païennes, Paris, Les Belles Lettres, $2002^{2}$ ), présente aussi de remarquables originalités. Commençons par les convergences. Dans le sillage de Turcan (op. cit. p. 26, 28-30 \& 63), ES (p. 25$26,41 \& 45-46$ ) insiste sur la finalité, tout à la fois principale et cachée, de Firmicus : se concilier la bienveillance des empereurs en montrant sa détermination pour la répression des cultes païens poursuivie par Constant et Constance II. Le pamphlétaire, qui avait lié son nom à l'œuvre astrologique Mathesis, après sa conversion au christianisme voulait montrer l'ardeur de son adhésion à la nouvelle religion, si fortement soutenue par les deux empereurs. ES ne néglige pas (p. 29 \& 39) les finalités déclarées par Firmicus : la volonté de conduire les païens à la conversion et la nécessité pour l'Empire de se garantir la faveur de Dieu en abandonnant de tels cultes; toutefois, comme Turcan, il les considère comme des enjeux secondaires. Cette interprétation repose sur la difficulté de penser que les interventions de Constant et Constance II contre les cultes païens aient pour origine le pamphlet de Firmicus.

ES (p. 39-43) suit Turcan aussi dans l'identification de la structure de l'oeuvre. Il distingue deux sections principales : la première consacrée à la présentation des religions païennes comme phénomènes de divinisation de réalités non divines, tels les quatre éléments (ch. I-V), les astres (ch. VIVIII), des hommes (ch. IX-XII) et des noms (ch. XIII-XVII); la deuxième concernant la démonstration de l'origine diabolique des mystères païens et la manifestation de leurs formules secrètes. L'espèce de phénoménologie apologétique des religions que Firmicus propose dans la première section se signale - affirme justement ES (p. 55), d'accord avec Turcan (op. cit. p. 50, n. 4) - comme un trait original par rapport aux sources païennes, et surtout chrétiennes, que le pamphlétaire semble généralement reprendre sans esprit novateur (p. 50-57).

Les enjeux de Firmicus sont mis en valeur par des hypothèses sur la situation politique de l'époque, originales par rapport au travail de Turcan. On notera comme spécialement intéressante la proposition de voir dans une phrase firmicienne (De errore XXIX, 2 : nec filio iubet parci nec fratri et per amata membra gladium vindicem ducit), qui trouve un écho dans une disposition du Code Théodosien (XVI, 10, 4 : si quis aliquid huiusmodi perpetraverit, gladio ultore sternatur) une allusion aux meurtres accomplis dans la descendance de Constantin après sa mort (p. 25). Considérant que cette loi peut dater de 346, le savant avance l'hypothèse que la composition du De errore lui serait contemporaine. Nouvelle aussi est la mise en avant d'une piste de recherche capable d'expliquer l'absence, dans le pam- 
phlet, de toute référence aux controverses doctrinales entre les Nicéens et les Ariens (p. 21). ES (p. 21-23) rappelle que les luttes pour la succession de Constantin qui divisèrent Constant et Constance II - le premier défenseur des catholiques et le second arien - commencèrent à s'apaiser dans les années quarante. A ce propos, il mentionne notamment le concile de Serdique en 343 et le retour, en 346, d'Athanase sur son siège épiscopal d'Alexandrie, d'où il avait été chassé par l'arien Constance II. Pour ES, le De errore entrerait dans les tentatives pour apaiser le climat d'hostilité, une visée qui aurait été facilitée par la possibilité, pour les deux empereurs, de combattre ensemble les cultes païens. ES se fonde, entre autres (p. 18), sur un papyrus magique copte (voir E. Sanzi, Magia e culti orientali IV. Tra maghi e sacerdoti : invocazioni di aiuto e richieste di successo nel mondo imperiale romano, in Ugo Criscuolo (éd.), Forme della cultura nella Tarda Antichità II. Atti del VI Convegno AST (Napoli - S. Maria Capua Vetere, 29 settembre - 2 ottobre 2003), Koinonia, 30-31, 2006-2007, p. 213-214 [je remercie ES de m'avoir communiqué son manuscrit]), pour confirmer que les lois des deux empereurs n'accomplirent pas leur finalité d'éliminer complètement les cultes païens.

L'appartenance d'ES à l'école historico-religieuse fondée par Bianchi se montre tout au long des notes qui fournissent un commentaire aussi bien synthétique que ponctuel. Il suffira ici de souligner deux exemples particulièrement représentatifs. A Firmicus qui, tout en dénonçant le scandale des actions immorales attribuées aux divinités païennes, rapporte les récits concernant Adonis (IX, 1-2), ES (p. 107, n. 68) ajoute une précision importante : conformément à une position bien développée dans les travaux de Bianchi et de ses disciples à l'égard de ce dieu (et d'analogues divinités « in vicenda ») il est inopportun d'employer pour Adonis la notion de résurrection. Sur ce point, le savant se distingue de Turcan (p. 46) qui, à propos d'une divinité soumise à des vicissitudes analogues comme Osiris, parle de « dieu mort et ressuscité ou remembré ». ES indique que la condition d'Adonis est plutôt celle de l'alternance : il descend dans l'Hadès pour y revenir, après un retour temporaire sur la terre. En deuxième lieu, commentant la formule divine " né de la pierre » $(\mathrm{XX}, 1)$ qui se réfère à la modalité de la naissance de Mithra, le savant (p. 142, n. 155) rappelle la raison de l'appellatif petrogenitus, puis insiste sur la nécessité de ne pas oublier la composante sotériologique du mithriacisme, qui présenterait non seulement un caractère intra-mondain, mais aussi une dimension eschatologique. Il s'agit d'une interprétation, valorisée là encore par Bianchi et son école (voir par exemple U. Bianchi, «Documento finale del seminario » \& «Epilegomena », in ID. [éd.], Mysteria Mythrae. Atti del Seminario Internazionale su «La specificità storico-religiosa dei Misteri di Mithra, con particolare riferimento alle fonti documentarie di Roma e Ostia ", Roma e Ostia, 28-31 Marzo 1978, Leiden-Rome, BrillEdizioni dell'Ateneo \& Bizzarri, 1979, p. XV \& 873-879; cf. récemment E. Sanzi, "The History of Religions in Italy during the Past Century. Raffaele Pettazzoni and Ugo Bianchi », Annals of the Sergiu Al George Institute, 9-11, 2000-2002, p. 242-243), mais sur laquelle le débat reste 
ouvert. D'ailleurs, Turcan, dans le compte rendu qu'il a donné dans cette revue (Revue de l'histoire des religions, 223, 2006, p. 229-231) d'un précédent volume de ES (I culti orientali nell'impero romano. Un'antologia di fonti, Cosenza, Giordano, 2003), ne s'est pas montré convaincu par cette dimension eschatologique.

Nous conclurons sur un troisième passage du De errore (XXII-XXIV, notamment XXII, 1) qui nous permet de montrer comment la mise à disposition de l'œuvre auprès d'un large public savant est d'autant plus précieuse que le pamphlet ne manque pas d'originalité discursive. Firmicus évoque un rituel nocturne de lamentation consacré au simulacre gisant d'un dieu dont l'identité n'est pas explicitée. S'interrogeant sur l'identification de la divinité, ES (p. 151, n. 165) suit Turcan, en estimant que l'hypothèse la plus convaincante est Osiris. Cette interprétation semble confirmée non seulement par la possible allusion aux membres dispersés du dieu qui sont recomposés pendant le rite (XXII, 3), mais aussi par une comparaison de la formule sacerdotale avec un fameux passage de Plutarque dans le De Iside et Osiride 27 (cf. l'intervention de U. Bianchi dans la discussion de Jean Pépin, «Réactions du christianisme latin à la sotériologie métroaque ", in U. Bianchi, M.J. Vermaseren (éds), La soteriologia dei culti orientali nell'Impero romano, Leiden, Brill, 1981, p. 273-274). À cette note historico-religieuse, nous proposons d'ajouter l'emploi de aliud se référant à l'unguentum qu'un prêtre impose sur la gorge des fidèles en les invitant à se livrer à l'espérance de la libération de leurs peines (XXII, 1 et 4 ; notamment XXIII, 1). De même que, dans le cas de Mithra (XX, 1-6, en part. XX, 1; cf. aussi II, 5 [alia est aqua, $=1$ 'eau du baptême] \& XVIII, 2 [alius est cibus [...] alius est cibus [...] alius est cibus $[\ldots],=1$ 'eucharistie), Firmicus avait rappelé, en martelant l'adjectif alius, que le véritable lapis n'est pas celui dont est né le dieu, mais le Christ, ici, l'auteur souligne que l'onguent du rite nocturne ne donne pas de salut : il y a un autre onguent salutaire, celui de l'onction baptismale. L'emploi d'alius pour exprimer l'opposition, traditionnelle dès les premiers apologistes (par ex., Justin, Dial. c. Tryph., 70, 1), entre les rites chrétiens et leur contrefaçon démoniaque nous semble une originalité de Firmicus. Par le biais de cette modalité rhétorique, l'auteur du pamphlet met à l'œuvre quasiment un retournement sémantique visant à exhorter au refus de la fausse signification païenne pour accueillir le véritable sens chrétien.

Grâce à ce volume, on peut désormais apprécier plus finement un pamphlet qui est important, surtout, comme attestation de certains hapax au sujet des cultes orientaux, mais qui ne manque pas d'originalité dans la structure et la mise en discours.

Giovanni TosetTI, Université de Messine. 